\title{
Natural Convection in a Partially Heated and Cooled Square Enclosure Containing a Diamond Shaped Heated Block
}

\author{
Kakali Chowdhury ${ }^{1}$, Abdul Alim², Murad Hossen ${ }^{1}$ \\ ${ }^{1}$ Department of Electrical \& Computer Engineering, Presidency University, Banani, Dhaka, Bangladesh \\ ${ }^{2}$ Department of Mathematics, Bangladesh University of Engineering \& Technology, Dhaka, Bangladesh \\ Email address: \\ kakalishome@yahoo.com (K. Chowdhury), maalim@math.buet.ac.bd (A. Alim), hossenm@pu.edu.bd (M. Hossen) \\ To cite this article: \\ Kakali Chowdhury, Abdul Alim, Murad Hossen. Natural Convection in a Partially Heated and Cooled Square Enclosure Containing a \\ Diamond Shaped Heated Block. International Journal of Fluid Mechanics \& Thermal Sciences. Vol. 6, No. 1, 2019, pp. 1-8. \\ doi: $10.11648 /$ j.ijfmts.20200601.11
}

Received: November 20, 2019; Accepted: December 11, 2019; Published: January 6, 2020

\begin{abstract}
Finite element method is used to solve the two dimensional governing mass, momentum and energy equations for steady state, natural convection problem inside a square enclosure. The enclosure consists of adiabatic vertical walls, heated middle part of bottom wall and the cold (top wall and the rest part of bottom wall) walls and a uniformly heated diamond shaped solid body located somewhere inside the enclosure. The aim of this study is to describe the effect of different sizes and positions of diamond shaped heated block on natural convection. The investigations are conducted for different values of Rayleigh number $(R a)$, block length $(l)$ and location of block center $\left(C_{x}, C_{y}\right)$ inside the enclosure by using COMSOL multiphysics. Various results such as streamlines, isotherms, heat transfer rate in terms of the average Nusselt number and average fluid temperature inside the enclosure are presented for different parameters. The results indicate that the average Nusselt number at the heated surface and average temperature of the fluid inside the enclosure are strongly dependent on the configuration of the system under different geometrical and physical conditions. The average Nusselt number decreases with the increasing value of block size and increases in the free convection dominated region, it is maximum for $R a=10^{6}$ and minimum for $R a=10^{3}$. Block size also has significant effect on thermal fields. Average temperature increases with the increasing value of heated block.
\end{abstract}

Keywords: Natural Convection, Finite Element Method, Diamond Shaped Block, COMSOL Multiphysics

\section{Introduction}

Natural convection is an essential mechanism of heat transfer in modern technology due to its wide applications. Various numerical and experimental investigations are done without and with block in the various shaped enclosure because these geometries have practical applications in engineering and industrial fields. Some mentionable fields are cooling of electronic devices, air conditioning, thermal design of building, design of solar collectors, chemical processing equipment, drying technologies etc. Literature review shows that various investigations are done on the mechanism of natural convection in a square enclosure containing various fluids with different geometrical parameters and boundary conditions. Ramonu et al. [1] analyzed natural convection of air in a rectangular cavity with partially heated and cooled side walls. They showed that the Rayleigh number drastically affects the flow profile and heat transfer behavior within the cavity. They also showed that for low Rayleigh numbers, natural convection reduces and heat transfer by conduction is predominant in the cavity. Parvin and Nasrin [2] analyzed the flow and heat transfer characteristics for MHD free convection in an enclosure with a heated block. They showed that the free convection parameter $R a$ and the diameter of circular body have significant effect on the flow and temperature fields. Chowdhury et al. [3] investigated the natural convection in porous triangular enclosure with a circular block in presence of heat generation. They showed that the fluid flow and temperature field strongly depend on the presence of the circular body and the average Nusselt number significantly worse with increasing both heat generation and size of the block. House et al. [4] investigated the effect of a centered, square heat conducting body on natural convection in a 
vertical enclosure. They showed that heat transfer across the cavity enhanced or reduced by a body with a thermal conductivity ratio less or greater than unity. Kandaswamy et al. [5] investigated natural convection in a square cavity in presence of heated plate. They showed that for the increasing value of $G r$ heat transfer rate increases in both vertical and horizontal situation. As the aspect ratio of heated thin plate increases the heat transfer also increases. Mousa [6] investigated the modeling of laminar buoyancy convection in a square cavity containing an block. They found that in case of low Rayleigh numbers $\left(10^{2}-10^{4}\right)$, the rate of heat transfer decreases when the aspect ratio of the adiabatic square block increases. In case of relatively high Rayleigh numbers $\left(10^{5}-\right.$ $\left.10^{6}\right)$, the maximum heat transfer rate increases when the aspect ratio of the adiabatic square block increases. Rahman et al [7] studied numerically the mixed convection in a square cavity with a heat conducting square cylinder at different locations. They showed that the flow field and temperature distributions inside the cavity are strongly dependent on the Richardson numbers and the position of the inner cylinder. Saleh et al. [8] investigated the natural convection heat transfer in a nanofluid-filled trapezoidal enclosure. They found that the structure of fluid flow within the enclosure depends upon Grashof number, inclination angle of slopping wall and nanoparticles concentration and type. Uddin et al. [9] investigated the natural convection flows in a trapezoidal enclosure with isoflux heating from below. They showed that the average Nusselt number increases with the increase of Rayleigh number and the effect of the sidewall inclination angle on heat transfer is significantly reduced at higher Rayleigh number. Roy and Basak [10] investigated finite element analysis of natural convection flows in a square cavity with non-uniformly heated wall (s). They found that in case of non-uniform heating the heat transfer rate is minimum at the edges of the heated walls and it reaches its maximum value at the center of both the heated walls. Billah et al [11] investigated simulation of MHD mixed convection heat transfer enhancement in a double lid driven obstructed enclosure. They found that the heat generating block size has a significant influence on the flow and thermal fields in the cavity. Oztop et al [12] investigated MHD mixed convection in a lid driven cavity with corner heater. They showed that dimensions of heater plays important role for natural convection dominant regime. Thermal boundary layer becomes higher with increasing of Hartmann number and isotherms fit with the corner. Basak et al. [13] investigated finite element based heatline approach to study mixed convection in a porous square cavity with various wall thermal boundary conditions. They found that at low Darcy number, the heatlines are smooth and parallel for all the $G r$, $\mathrm{Pr}$ and Re. Rehena Nasrin [14] investigated mixed magnetoconvection in a lid driven cavity with a sinusoidal wavy wall and a central heat conducting body. she found that the influence of $\mathrm{Ha}$ does not affect significantly the thermal current activities. But the flow pattern changes dramatically owing to the hindrance of the imposed magnetic field. Kabir et al. [15] investigated effects of viscous dissipation on MHD natural convection flow along a vertical wavy surface. They found that velocity, temperature and the frictional force at the wall enhance due the higher valuesof the Eckert number $E c$, while the rate of heat transfer reduces for the greater values of Eckert number. The effects of $E c$ on skin friction and on the rate of heat transfer are more significant than that on the velocity and temperature.

On the basis of the literature review it seems that, till date the numerical investigations on partially heated enclosures with heated block of different shapes based on streamlines and isotherms are limited to analyse. No work was done on natural convection in a partially heated and cooled square enclosure containing a diamond shaped heated block. Therefore, on the basis of its practical application in the engineering and industrial field, this topic requires further analysis.

The objective of the present study is to examine the effect of different sizes and positions of diamond shaped heated block on natural convection in a partially heated and cooled square enclosure. The investigations are carried out for various parameters such as Rayleigh number $(R a)$, block length (l) and location of block center $\left(C_{x}, C_{y}\right)$ inside the enclosure by using COMSOL multiphysics 5.4.

\section{Physical Model}

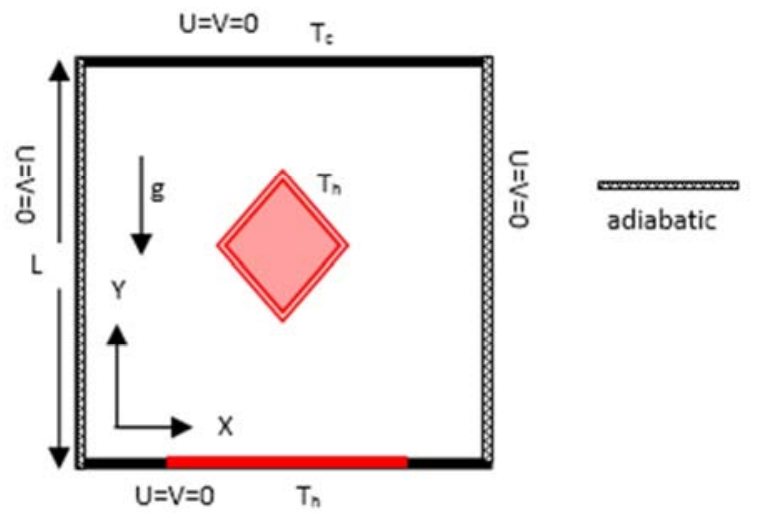

Figure 1. Schematic view of the enclosure considered in present study.

\subsection{Mathematical Formulation}

The motion of a fluid within a partially heated square enclosure of length $L$ is considered in this study. It is assumed that the flow is steady, two-dimensional, laminar, and incompressible and there is no viscous dissipation. The gravity force acts in the vertically downward direction, fluid properties are constant and fluid density variations are neglected except in the buoyancy term (Boussinesq approximation) and radiation effect is neglected. Under the usual Boussinesq approximation, the governing equations for the present problem can be described in dimensionless form by the following equations.

$$
\frac{\partial U}{\partial X}+\frac{\partial V}{\partial Y}=0
$$




$$
\begin{gathered}
U \frac{\partial U}{\partial X}+V \frac{\partial U}{\partial Y}=-\frac{\partial P}{\partial X}+\operatorname{Pr}\left(\frac{\partial^{2} U}{\partial X^{2}}+\frac{\partial^{2} U}{\partial Y^{2}}\right) \\
U \frac{\partial V}{\partial X}+V \frac{\partial V}{\partial Y}=-\frac{\partial P}{\partial Y}+\operatorname{Pr}\left(\frac{\partial^{2} V}{\partial X^{2}}+\frac{\partial^{2} V}{\partial Y^{2}}\right)+R a \operatorname{Pr} \theta \\
X=\frac{x}{L}, \quad Y=\frac{y}{L}, \quad U=\frac{u}{U_{0}}, \\
\text { The governing parameters in the preceding equations are } \\
\text { the Rayleigh number } R a=\frac{g \beta\left(T_{h}-T_{c}\right) L^{3}}{v \alpha} \text { and Prandtl }
\end{gathered}
$$
number $\operatorname{Pr}=\frac{v}{\alpha}$. The associated dimensionless boundary conditions are as follows: $P=0$; at all boundaries $U=V=0$; at the square enclosure walls $\theta=0$; at the cooled walls $\theta=1$; at the heated walls $\frac{\partial \theta}{\partial Y}=0$; at the vertical walls and at the diamond shaped block surface $U=V=0, \quad \theta=1$.

The heat transfer coefficient in terms of the local Nusselt number is defined by $N u=-\frac{\partial \theta}{\partial \eta}$ where $\eta$ denotes the normal direction on a plane and the bulk average temperature in the enclosure is defined as $\theta_{a v}=\int \frac{\theta}{S} d S$.

\subsection{Numerical Technique}

The numerical procedure used in this work is based on the Galerkin weighted residual method of finite element formulation. The application of this technique is well described by Taylor and Hood and Dechaumphai. In this method, the solution domain is discretized into finite element

$$
U \frac{\partial \theta}{\partial X}+V \frac{\partial \theta}{\partial Y}=\left(\frac{\partial^{2} \theta}{\partial X^{2}}+\frac{\partial^{2} \theta}{\partial Y^{2}}\right)
$$

The dimensionless variables are defined as:

$V=\frac{v}{U_{0}}, \quad P=\frac{p}{\rho U_{0}^{2}}, \quad \theta=\frac{T-T_{c}}{T_{h}-T_{c}}$

meshes, which are composed of non-uniform triangular elements. Then the nonlinear governing partial differential equations (i.e. mass, momentum and energy equations) are transferred into a system of integral equations by applying Galerkin Residual method. The integration involved in each term of these equations is performed by using Gauss's quadrature method. The nonlinear algebraic equations so obtained are modified by imposition of boundary conditions. These modified nonlinear equations are transferred into linear algebraic equations by Newton-Raphson iteration. Finally, these linear equations are solved by using Triangular Factorization method.

\subsection{Numerical Validation}

To validate the present numerical code, the results for natural convection flow around a heated diamond shaped block placed somewhere in the enclosure have been compared with those obtained by Ramonu et al. The comparison of the results obtained from present code with those of Ramonu et al. is demonstrated for two different Rayleigh numbers $R a=10^{5}$ and $R a=10^{7}$ at $\operatorname{Pr}=0.71$ in figure 2 . As seen from these figures the obtained results show very good agreement.

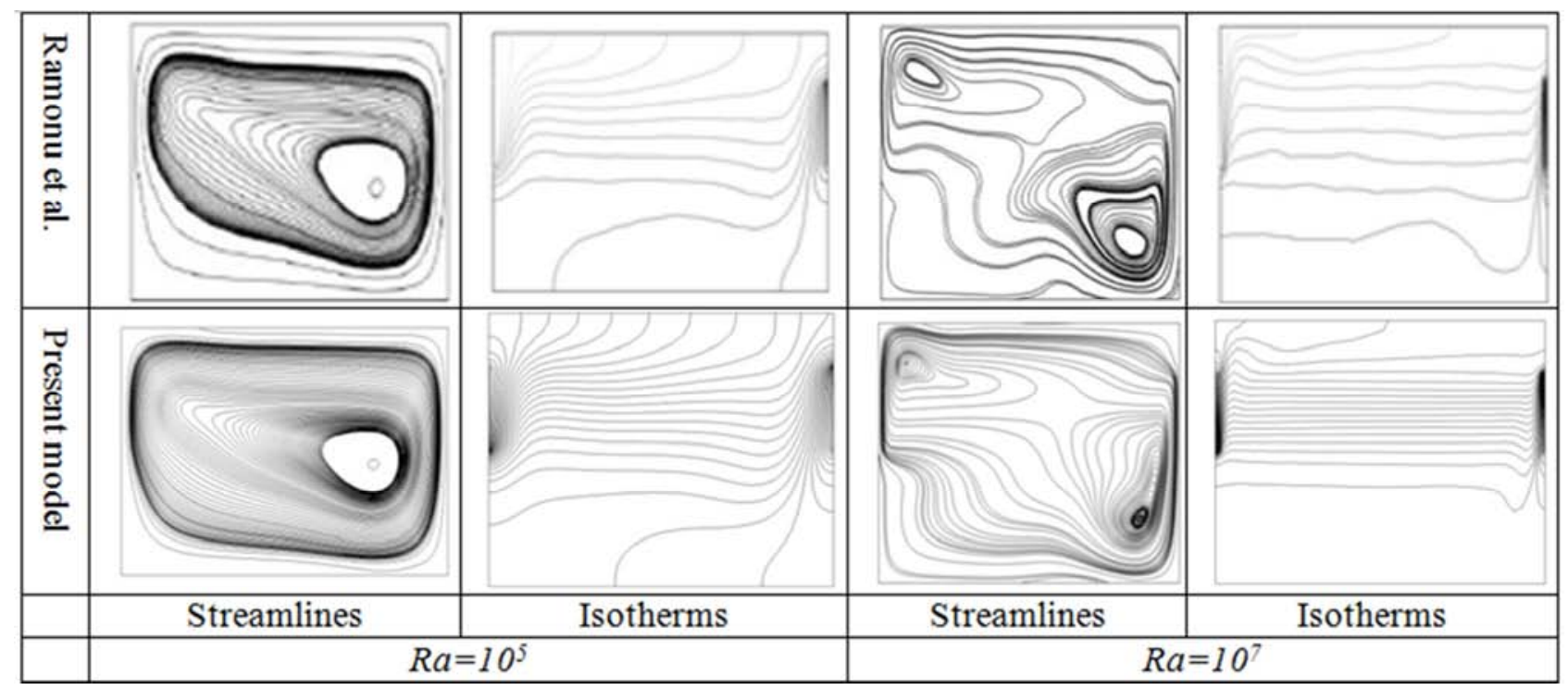

Figure 2. Comparison of streamlines and isotherms between present model (lower) and the model of Ramonu et al. (upper) at Pr=0.71. 


\section{Results and Discussion}

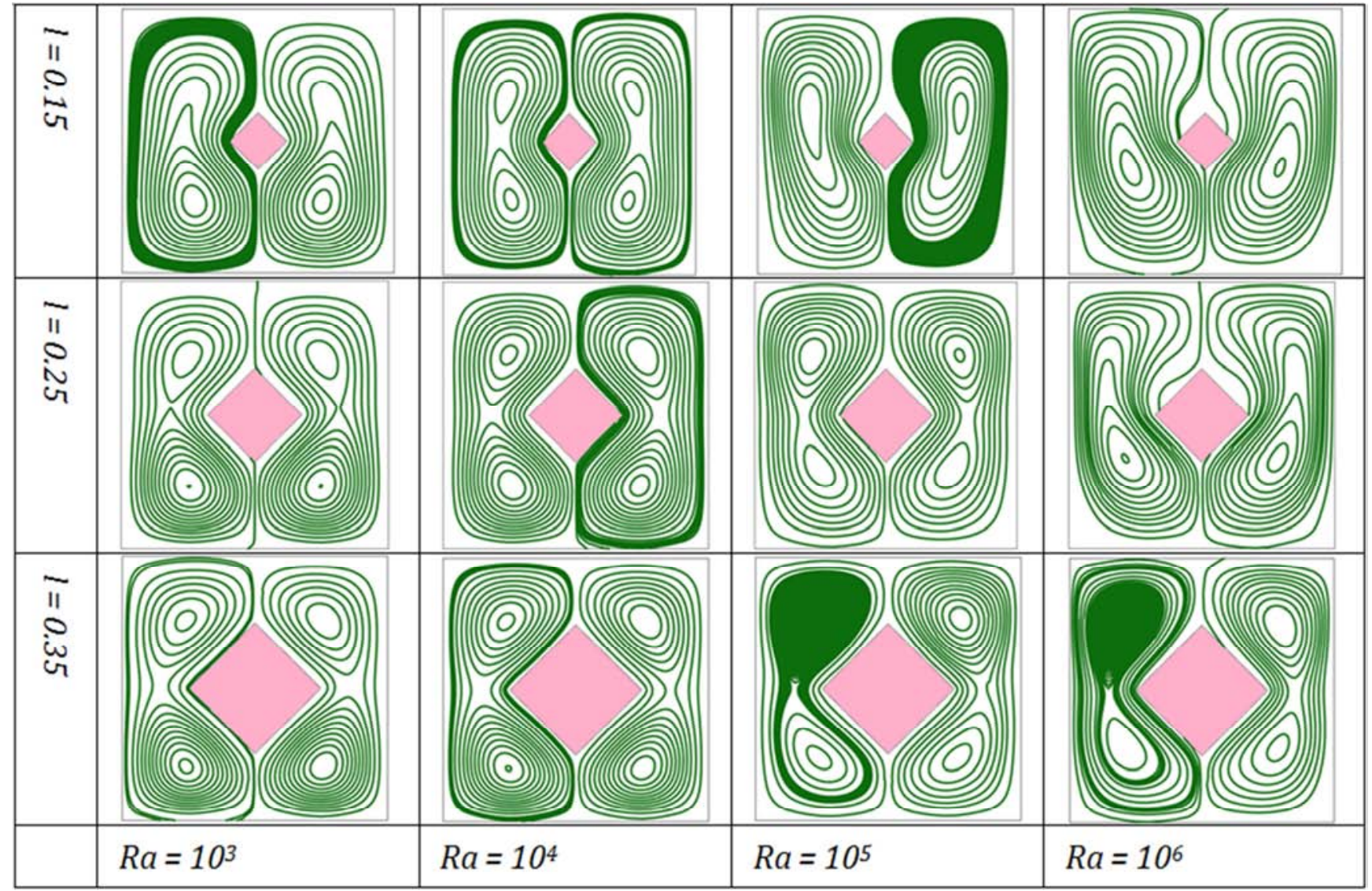

Figure 3. Streamlines for different values of Ra and $l$ with $P r=0.71$ and centre at $(0.5,0.5)$.

A numerical study has been performed through finite element method to analyze the laminar natural convection heat transfer and fluid flow in an enclosure containing a diamond shaped heated block. Effects of the Rayleigh number $(R a)$, block length $(l)$, and the center position of heated block $\left(C_{x}, C_{y}\right)$ on heat transfer and fluid flow have been analyzed. The result is presented in two sections. The first section has focused on flow and temperature fields, which contains the streamlines and isotherms for different cases. Heat transfer including average Nusselt number at heated surface and average fluid temperature in the enclosure has been discussed in the following section. In this investigation, the range of $R a$ varies from $R a=10^{3}$ to $R a=10^{6}$ and the range of $l$ varies from $l=0.15$ to $l=0.35$. Centre position $\left(C_{x}, C_{y}\right)$ of heated block are considered at $(0.25,0.35),(0.75,0.35),(0.25,0.75)$ and $(0.75,0.75)$ while the Prandtl number $\operatorname{Pr}$ is kept fixed at 0.71 . Being heated the fluid rises up from the central region as a result of buoyancy forces, created by the temperature difference of heated and cold part of the enclosure and then it flows down along the vertical walls forming two symmetric vortices with clockwise (right vortex) and anticlockwise (left vortex) rotations inside the enclosure. The direction of the vortices is shown in figure 4. The influence of Rayleigh number $R a$ (from $R a=10^{3}$ to $10^{6}$ ) and block length $l$ (from $l=0.15$ to 0.35 ) on heat transfer for the present configuration at $\operatorname{Pr}=0.71$ and center position $\left(C_{x}, C_{y}\right)$ at $(0.5$, 0.5 ) has been demonstrated in Figure 3. At $R a=10^{3}$, viscous forces are more dominant than the buoyancy forces and hence, heat transfer is essentially conduction dominated and the circulation is so weak that the shape of the streamline tends to follow the geometry of the enclosure. With the increasing value of $\mathrm{Ra}$, the core of the vortices are more circulating indicating significant increase of the intensity of convection. Figure 4 depicts the direction of the vortices for $R a=10^{3}$ and $R a=10^{6}$ where $l=0.15, \operatorname{Pr}=0.71$ and center position $\left(C_{x}, C_{y}\right)$ at $(0.5,0.5)$.

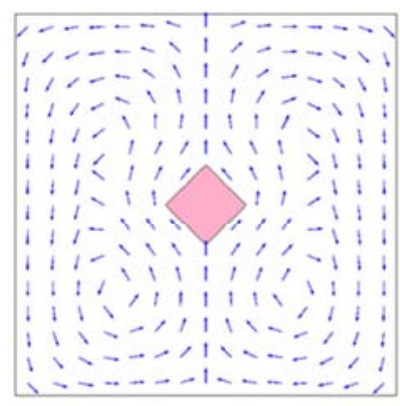

$\mathrm{Ra}=10^{3}$

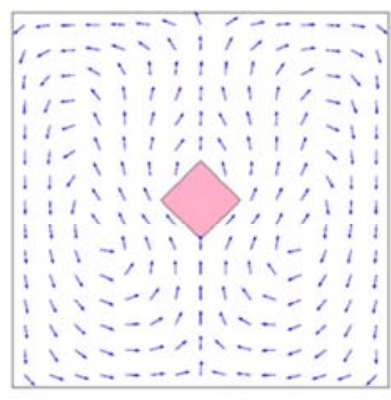

$\mathrm{Ra}=10^{6}$
Figure 4. Arrow surface at $l=0.15, P r=0.71$ and diamond center at $(0.5$, $0.5)$. 


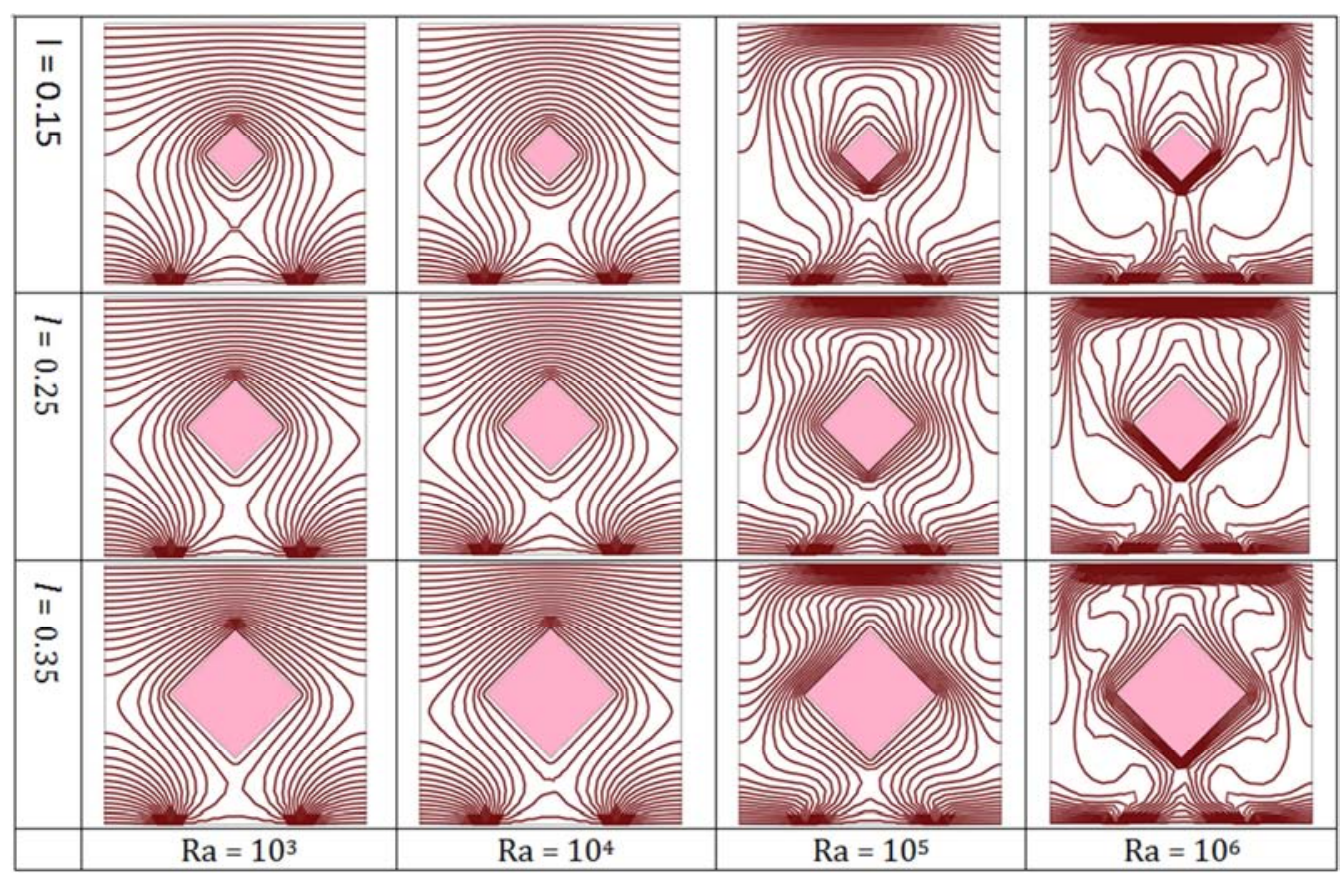

Figure 5. Isotherms for different values of Ra and $l$ with $\mathrm{Pr}=0.71$ and diamond center at $(0.5,0.5)$.

The influence of Rayleigh number $R a$ (from $R a=10^{3}$ to $10^{6}$ ) and block length $l$ (from $l=0.15$ to 0.35 ) on temperature field for the present configuration at $\operatorname{Pr}=0.71$ and center position $\left(C_{x}, C_{y}\right)$ at $(0.5,0.5)$ has been demonstrated in Figure 5. At $R a=10^{3}$ the conduction dominant heat transfer modes are available and the isothermal lines are almost parallel. At $R a=10^{4}$ the characteristics of the isotherms are not so different than that of $\mathrm{Ra}=10^{3}$. But with the increasing value of $R a$ due to the initiation of convection, isotherms started to distort and at $R a=10^{6}$ the isotherms are significantly distorted and pushed near the cold walls. In this regime, due to the increase of circulation intensity the distorted isothermal lines form thermal boundary layer near the cold walls. At $R a=10^{5}$ and $10^{6}$ these lines accumulate near the upper horizontal wall creating a plume just above the diamond shaped heated block and it divides the bottom wall in three parts.

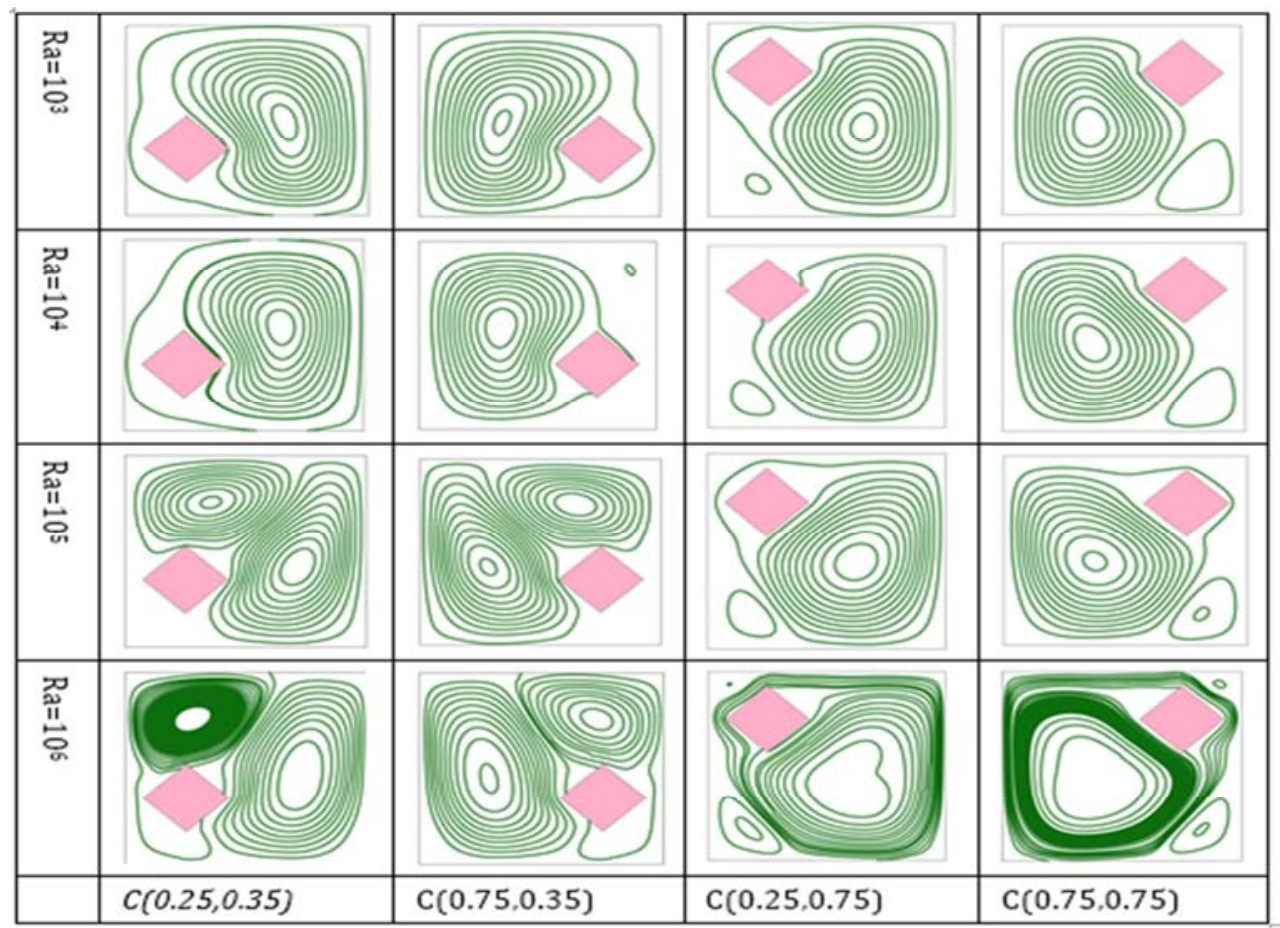

Figure 6. Streamlines for different values of Ra and different position of block center with Pr=0.71 and l=0.25. 


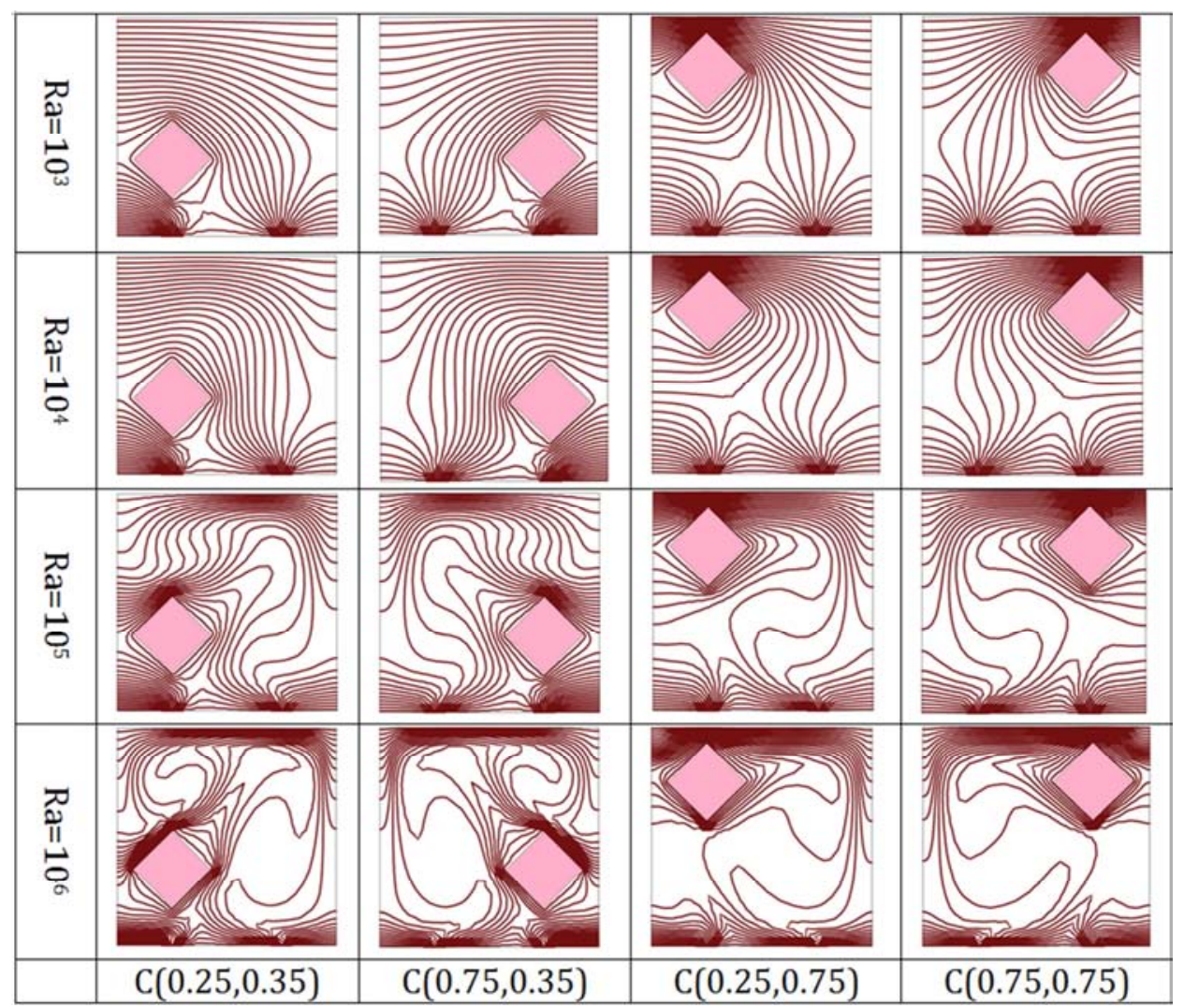

Figure 7. Isotherms for different values of Ra and different position of block center with $P r=0.71$ and $l=0.25$.

The isothermal lines also create another plume just above the heated part of horizontal wall. The influence of Rayleigh number $R a$ (from $R a=10^{3}$ to $10^{6}$ ) and block center $\left(C_{x}, C_{y}\right.$ ) at different positions on streamlines has been demonstrated in figure 6. Here block length $l$ is considered as 0.25 and $P r$ is kept fixed as 0.71 . At $R a=10^{3}$ where the fluid is almost conduction dominated, the streamlines have taken the shape of the geometric figure creating a large vortex in the opposite side of the block. With the increasing value of $R a$ the circulation rises up and the streamlines are divided in two vortices. The position of the block has insignificant effect on the streamlines.

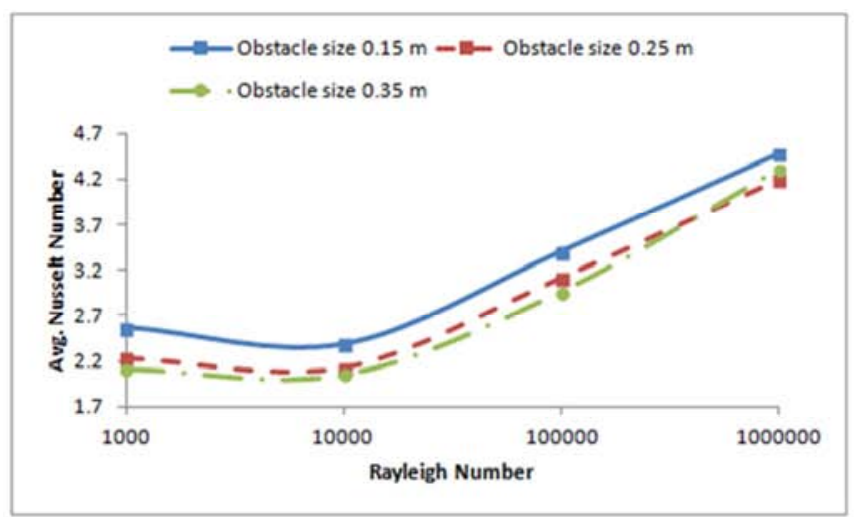

The influence of Rayleigh number $R a$ (from $R a=10^{3}$ to $10^{6}$ ) and block center $\left(C_{x}, C_{y}\right)$ at different positions on isotherms has been demonstrated in figure 7 . Here block length $l$ is considered as 0.25 and $\operatorname{Pr}$ is kept fixed as 0.71 . At $R a=10^{3}$ and $R a=10^{4}$ the distortion of the isotherms are negligible because heat transfer in this region is mostly conduction dominated. With the increasing value of $R a$, the intensity of convection increases and as before the isotherms are significantly distorted and pushed near the cold walls. As a result near the horizontal walls boundary layers are created.

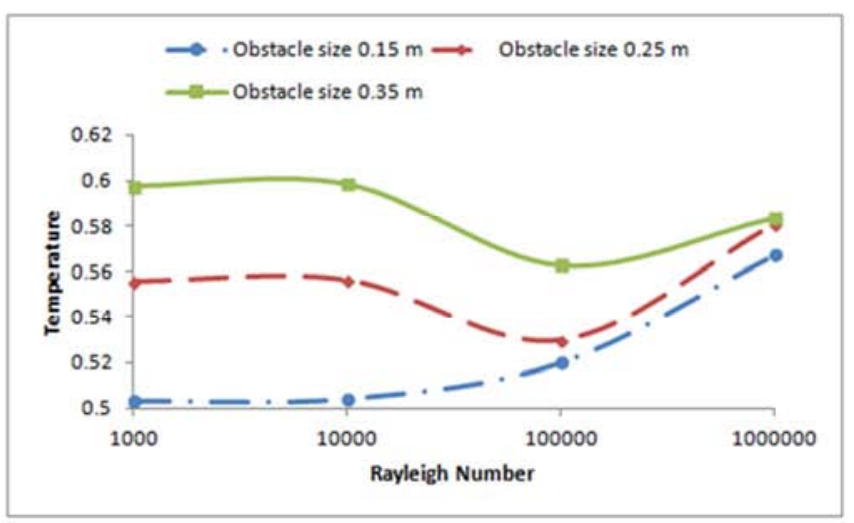

Figure 8. Effect of block on (a) average Nusselt number (b) average temperature with $\mathrm{Pr}=0.71$ and center at $(0.5,0.5)$.

In order to evaluate how the block size effects on the heat transfer, the average Nusselt number is plotted as a function of Rayleigh number as shown in Figure 8 (a) for three different block sizes $(l=0.15,0.25$, and 0.35$)$ where $\operatorname{Pr}=0.71$ 
and block center at $(0.5,0.5)$ are kept fixed. It is observed that $N u$ decreases with the increasing value of block length or area. For all values of block size $N u$ decreases slightly from $R a=10^{3}$ to $R a=10^{4}$ that is in the conduction dominated heat transfer region and $\mathrm{Nu}$ starts to increase from $R a=10^{4}$ that is where free convection initiates. From $R a=10^{4}$ to $R a=10^{6}$ heat transfer gradually increases for all values of block size. Figure 8 (b) depicts the average temperature of fluid as a function of Rayleigh number $R a$ while $P r=0.71$ and block center at $(0.5,0.5)$ are kept fixed. It is observed that fluid temperature increases with the increasing value of block length. For all values of block length $l$, temperature increases almost linearly in the conduction dominated region and increases gradually in the pure free convection dominated region.

\section{Conclusion}

A numerical investigation is made for steady-state, incompressible, laminar natural convection flow in a square enclosure containing a heated diamond shaped block. Results are obtained for wide ranges of parameters Rayleigh number $(R a)$, dimensionless block length $(l)$ and the location of circular block $\left(C_{x}, C_{y}\right)$. The major findings have been summarized as follows:

a) The fluid flow and fluid temperature are strongly dependent on Rayleigh number. The value of average Nusselt number is maximum for $R a=10^{6}$ and minimum for $R a=10^{3}$.

b) Locations of the block have insignificant effect on fluid flow and thermal fields.

c) Block size has a significant effect on thermal fields. Average temperature increases with the increasing value of heated block.

d) The average Nusselt number $N u$ significantly decreases with the increasing value of block size but it increases in the pure free convection dominated region.

\section{Nomenclature}

$c_{p}$
$\mathrm{~g}$
$h$
$k$
$T$
$\Delta T$
$u, v$
$U, V$
$p$

specific heat at constant pressure gravitational acceleration $\left[\mathrm{ms}^{-2}\right]$ convective heat transfer coefficient $\left[\mathrm{Wm}^{-2} \mathrm{~K}^{-1}\right]$ thermal conductivity of fluid $\left[\mathrm{Wm}^{-1} \mathrm{~K}^{-1}\right]$ dimensional temperature $[K]$ dimensional temperature difference $[K]$ dimensional velocity components $\left[\mathrm{ms}^{-1}\right]$ dimensionless velocity components dimensional pressure $\left[\mathrm{Nm}^{-2}\right]$

Greek symbols

$\begin{array}{ll}\alpha & \text { thermal diffusivity }\left[\mathrm{m}^{2} \mathrm{~s}^{-1}\right] \\ \beta & \text { thermal expansion coefficient }\left[\mathrm{K}^{-1}\right] \\ v & \text { kinematic viscosity }\left[\mathrm{m}^{2} \mathrm{~s}^{-1}\right] \\ \theta & \text { non dimensional temperature } \\ \rho & \text { density of the fluid }\left[\mathrm{kgm}^{-3}\right]\end{array}$

\section{References}

[1] Ramonu O. J., Afolabi S. I., Akinyemi T. O. 2018. Natural convection of air in a rectangular cavity with partially heated and cooled side walls. ResearchGate.

[2] Parvin S., Nasrin R. 2011. Analysis of the flow and heat transfer characteristics for MHD free convection in an enclosure with a heated obstacle. Nonlinear Analysis: Modeling and Control, Vol. 16, No. 1, 89-99.

[3] Chowdhury, R., Khan, M. A. H., Siddiki, M. N. A. A., 2015. Natural Convection in Porous Triangular Enclosure with a Circular Obstacle in Presence of Heat Generation. American Journal of Applied Mathematics, 3 (2): pp. 51-58. Dechaumphai, P., 1999. Finite Element Method in Engineering, $2^{\text {nd }}$ ed. Chulalongkorn University Press, Bangkok.

$\begin{array}{ll}x, y & \text { cartesian coordinates } \\ X, Y & \text { dimensionless cartesian coordinates } \\ \left(C_{x}, C_{y}\right) & \text { diamond shaped block centre } \\ N u & \text { average Nusselt number } \\ P & \text { dimensionless pressure } \\ P r & \text { Prandtl number } \\ R a & \text { Rayleigh number } \\ l & \text { diamond shaped block length }[\mathrm{m}] \\ \mathrm{L} & \text { Height of the enclosure [m] } \\ & \text { Subscripts } \\ c & \text { cold } \\ h & \text { heated }\end{array}$

[4] House, J. M., Beckermann, C., Smith, T. F., 1990. Effect of a centered conducting body on natural convection heat transfer in an enclosure, Numerical Heat Transfer A-Appl., 18, pp. 213-225.

[5] Kandaswamy, P., Lee, J., Hakeem A. K. A., 2007. Natural convection in a square cavity in presence of heated plate. Nonlinear Analysis: Modeling and Control, Vol. 12, No. 2, 203-212.

[6] Mousa, M. M. 2010. Modeling of laminar buoyancy convection in a square cavity containing an obstacle. Mathematics Subject Classification: 65M60, 76D05, 80A20.

[7] Rahman, M. M., Alim, M. A., Saha, S., Chowdhury, M. K., 2008. A numerical study of mixed convection in a square cavity with a heat conducting square cylinder at different locations. Journal of Mechanical Engineering, Vol. ME39, No. 2. 
[8] Saleh, H., Roslan, R., Hashim I., 2011. Natural convection heat transfer in a nanofluid-filled trapezoidal enclosure. International Journal of Heat and Mass Transfer. pp. 194-201, Issues 1-3, Vol. 54, Jan-2011.

[9] Uddin, H., Saha, S., Hasan, M. N., 2008. Natural convection flows in a trapezoidal enclosure with isoflux heating from below. Proceedings of the $12^{\text {th }}$ Annual Paper Meet, Paper ID: 182-EN08.

[10] Roy, S., Basak, T., 2005. Finite element analysis of natural convection flows in a square cavity with non-uniformly heated wall (s) International Journal of Engineering Science 43 (2005) 668-680.

[11] Billah, M. M., Rahman, M. M., Saidur, R., Hasanuzzaman, M. 2011. Simulation of MHD mixed convection heat transfer enhancement in a double lid driven obstructed enclosure. International Journal of Mechanical and Materials Engineering (IJMME). Vol. 6, No. 1, 18-30.
[12] Oztop, H. F., Al-Salem, K, Pop I., 2011. MHD mixed convection in a lid driven cavity with corner heater. International Journal of heat and mass transfer 54, 3494-3504.

[13] Basak, T., Predeep, P. V. K., Roy, S., Pop, I. 2011. Finite element based heatline approach to study mixed convection in a porous square cavity with various wall thermal boundary conditions. International Journal of heat and mass transfer. 54, 1706-1727.

[14] Nasrin, R. 2011. Mixed magnetoconvection in a lid driven cavity with a sinusoidal wavy wall and a central heat conducting body. Journal of naval architecture and marine engineering. DOI: 10.3329/jname.v8il.6793.

[15] Kabir, K. H., Alim, M. A., Andallah L. S. 2013. Effects of viscous dissipation on MHD natural convection flow along a vertical wavy surface. Journal of Theoritical and Applied Physics. Doi: 10.1186/2251-7235-7-31. 